\title{
S-R compatibility effects due to context-dependent spatial stimulus coding
}

\author{
BERNHARD HOMMEL and YVONNE LIPPA \\ Max Planck Institute for Psychological Research, Munich, Germany
}

\begin{abstract}
Responses are faster with spatial S-R correspondence than with noncorrespondence (spatial compatibility effect), even if stimulus location is irrelevant (Simon effect). In two experiments, we sought to determine whether stimuli located above and below a fixation point are coded as left and right (and thus affect the selection of left and right responses) if the visual context suggests such a coding. So, stimuli appeared on the left or right eye of a face's image that was tilted by $90^{\circ}$ to one side or the other (Experiment 1) or varied between upright and $45^{\circ}$ or $90^{\circ}$ tilting (Experiment 2). Whether stimulus location was relevant (Experiment 1) or not (Experiment 2), responses were faster with correspondence of (face-based) stimulus location and (egocentrically defined) response location, even if stimulus and response locations varied on physically orthogonal dimensions. This suggests that object-based spatial stimulus codes are formed automatically and thus influence the speed of response selection.
\end{abstract}

Some of the most remarkable demonstrations of the interplay between perception and action are provided by phenomena of stimulus-response ( $\mathrm{S}-\mathrm{R}$ ) compatibility. Among these, effects of spatial S-R compatibility are especially robust. If, for instance, subjects respond to left and right stimuli by pressing a left or right key, left responses are faster to left stimuli than to right stimuli, whereas right responses are faster to right stimuli than to left stimuli (e.g., Broadbent \& Gregory, 1962). That is, spatial S-R correspondence permits better performance than does noncorrespondence.

A particularly convincing manifestation of the robustness of spatial compatibility effects is that S-R correspondence affects choice reactions even with irrelevant stimulus position. If, for instance, subjects respond with a left or right key to a nonspatial attribute of a stimulus that appears randomly on the left or right side, responses are faster if stimulus and response positions correspond than if they do not (e.g., Simon, Hinrichs, \& Craft, 1970). This effect of irrelevant $\mathrm{S}-\mathrm{R}$ correspondence has come to be known as the Simon effect.

In most spatial compatibility tasks or Simon tasks, stimulus position can be defined as left or right relative to several possible spatial reference frames, such as retinal, egocentric, or environmental (for a discussion of reference frames see, e.g., Corballis, 1988; Hinton \&

We wish to thank Bettina Herborn for finding and preparing the context stimulus of Experiments 1 and 2 and for conducting Experiment 2, Benjamin Beyer for running Experiment 1, Andreas Schmidt for technical assistance, Heidi John for checking and improving the English, and Robert Proctor, Richard Schweickert, and an anonymous reviewer for constructive criticism and helpful suggestions. Address correspondence to either author, Max Planck Institute for Psychological Research, Leopoldstr. 24, D-80802 München, Germany (e-mail: hommel@, mpipf-muenchen.mpg.de or lippa@mpipf-muenchen.mpg.de).
Parsons, 1981; Palmer, 1989). That is, stimulus locationreference frame relationships are mostly confounded, so that it is not clear which frame is relevant for spatial stimulus coding. However, there is some evidence that spatial stimulus codes are formed relative to several reference frames. For example, Umiltà and Liotti (1987) showed that both stimulus side and relative position are coded and produce compatibility effects. Likewise, Lamberts, Tavernier, and d'Ydewalle (1992) found (additively combining) S-R correspondence effects related to stimulus side, visual hemifield, and relative position. Interestingly, these studies yielded comparable effects for spatial compatibility tasks and for Simon tasks. Stimulus position was irrelevant in the latter, suggesting that multiple spatial stimulus codes ${ }^{1}$ are formed automatically; that is, their formation is not due to, and does not depend on, the subject's intention or strategy, nor on the code's utility for performing the task.

The present study investigates whether visual stimuli are also coded relative to an object-based or intrinsic frame of reference (Hinton \& Parsons, 1981; Rock, 1973), if they are embedded in a larger visual context. Figure 1 shows the computer scan of a picture of Marilyn Monroe's face that served as such a context. ${ }^{2}$ The stimuli were solid circles appearing on - and thus temporarily covering - the face's left or right eye, from the subject's perspective. With an upright face, we would, of course, expect that stimuli covering the left and the right eye would be coded as left and right, thus producing a standard spatial S-R compatibility effect. However, what would happen if the face is tilted $90^{\circ}$ to the left or right, so that the stimuli now appear above and below the center of the face? If the visual context does not affect spatial stimulus coding, the standard compatibility effect should disappear, since there is no variation of stimulus position in terms of left and right relative to retinal, 


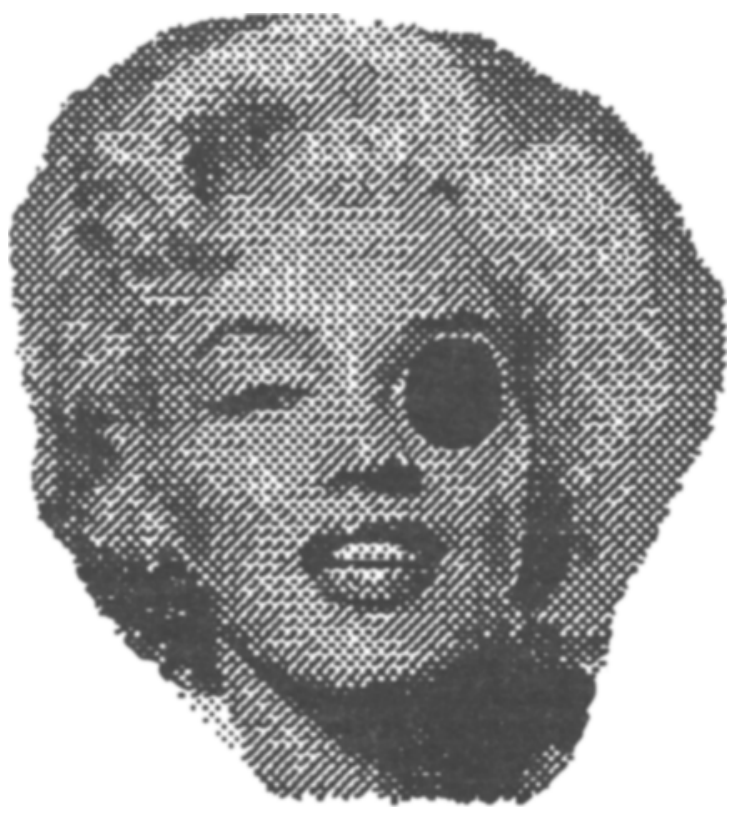

Figure 1. Computer-scanned pixel representation of the face of Marilyn Monroe, used as a context stimulus in Experiments 1 and 2, including a black stimulus covering the right eye.

egocentrical, or environmental reference frames. If, however, stimuli are coded with regard to face orientationhence, in intrinsic coordinates-a corresponding S-R compatibility effect should emerge. That is, even with the face tilted, left and right responses should be comparably faster if the stimulus appears on the face's left or right eye, respectively.

\section{EXPERIMENT 1}

In Experiment 1, we investigated context-dependent stimulus coding in a spatial compatibility task. Subjects responded to the vertical position of a black circle by pressing a left or right key. The circle appeared on one or the other eye of the face's image, which was tilted $90^{\circ}$ to the left or right. That is, with a left-tilted image, the bottom stimulus would be intrinsically coded as left and the top one as right, whereas with a right-tilted image, the opposite would be true. Performance was expected to be better with correspondence between context-defined stimulus position and relative response position (i.e., with eye-response correspondence) than with noncorrespondence. Moreover, we tested whether the orientation of the context stimulus (i.e., direction of tilting) would also affect response selection (i.e., produce an effect of orientation-response correspondence).

\section{Method}

Twenty-four adults were paid to participate in single sessions of about $15 \mathrm{~min}$. They reported having normal or corrected-to-normal vision and were naive as to the purpose of the experiment. The experiment was controlled by an Atari STE connected to an Atari SM 124 high-resolution $(640 \times 400$ pixels) monochrome monitor. Responses were made by pressing the right or left shift key of the computer keyboard with the corresponding index finger. From a viewing distance of approximately $60 \mathrm{~cm}$, the subject saw the face serving as context stimulus (see Figure 1) tilted $90^{\circ}$ to the left or right. The image measured $7.3^{\circ}$ in width (hairline-to-chin axis) and $6.7^{\circ}$ in height (ear-to-ear axis) and remained visible throughout the respective orientation block. The white fixation cross appeared on its pivot (i.e., on the bridge of the nose). The stimuli, solid black circles of $1^{\circ}$ in diameter, appeared $0.9^{\circ}$ above or below the fixation point, thus covering the left or the right eye.

Stimuli were introduced as top and bottom. Half of the subjects responded by pressing the left and the right key, respectively, whereas the other half received the opposite $\mathrm{S}-\mathrm{R}$ mapping. In each trial, the intertrial interval of $2,500 \mathrm{msec}$ was followed by a $150-\mathrm{msec}$ presentation of the fixation cross. Two hundred milliseconds later, the stimulus was presented and remained until either a response was given or $1 \mathrm{sec}$ had passed. Pressing the wrong key counted as an error, and trials with latencies exceeding $1 \mathrm{sec}$ were considered missing. Both kinds of trials were recorded and then repeated at a random position in the remainder of the block.

The subjects worked through 11 blocks ( 1 warm-up, 10 experimental) of 16 trials each. Each block had two halves, one for each face orientation. Per orientation, there were eight randomly intermixed trials, consisting of four replications for each of the two stimulus positions. Orientation always alternated between left and right, with the start orientation being balanced over subjects.

\section{Results and Discussion}

Missing trials $(<0.3 \%)$ were excluded from analysis. Mean reaction times (RTs) and percentages of error (PEs) were calculated for each subject as a function of spatial correspondence or noncorrespondence between eye and response and between face orientation and response (see Table 1 for group means), so that each individual $R T$ mean was based on 40 valid observations. An analysis of variance (ANOVA) of the RTs, with S-R mapping as a between-subjects factor, confirmed that responses were significantly faster with eye-response correspondence $(364 \mathrm{msec})$ than with noncorrespondence $(371 \mathrm{msec})[F(1,22)=6.46, p<.02]$. The main effects of mapping and orientation-response correspondence were far from significance $(p>.23$ and $p>.20$, respectively), as were the interaction terms $(p>.7)$. The error analysis did not reveal any significant result.

The presence of an eye-response correspondence effect demonstrates that the stimuli were coded as left and right, though no such coding could have resulted from referring to retinal, egocentrical, or environmental frames of reference. That is, the stimuli must have been coded relative to the structure of the visual contexthence, in intrinsic coordinates. Note, however, that the correspondence effect amounted to $7 \mathrm{msec}$ only, which is very little relative to the $50-100 \mathrm{msec}$ obtained in normal spatial compatibility tasks (e.g., Broadbent \& Gregory, 1962; Riggio, Gawryszewski, \& Umiltà, 1986; Umiltà \& Liotti, 1987). This suggests that the standard spatial compatibility effect cannot be attributed exclusively to intrinsically defined stimulus position. More likely, an intrinsic position code is only one of many spatial stimulus codes, referring to, for instance, egocentric or retinal spatial relations (see Lamberts et al., 1992). If so, compatibility effects would be largest if all relevant (i.e., response-related) stimulus codes corre- 
Table 1

Mean Reaction Times (RTs, in Milliseconds), Standard Deviations (SDs), and Percent Errors (PEs) in Experiment 1 as a Function of

Stimulus-Response Mapping, Orientation-Response

Correspondence (ORC), and Eye-Response

Correspondence (ERC)

\begin{tabular}{|c|c|c|c|c|c|c|c|}
\hline \multirow[b]{3}{*}{ ORC } & \multirow[b]{3}{*}{ ERC } & \multicolumn{6}{|c|}{ Mapping } \\
\hline & & \multicolumn{3}{|c|}{ Top-Left/Bottom-Right } & \multicolumn{3}{|c|}{ Top-Right/Bottom-Left } \\
\hline & & $\mathrm{RT}$ & $S D$ & $\mathrm{PE}$ & RT & $S D$ & $\mathrm{PE}$ \\
\hline+ & + & 381 & 72 & 2.4 & 352 & 39 & 3.0 \\
\hline+ & - & 386 & 72 & 2.0 & 360 & 54 & 2.4 \\
\hline- & + & 376 & 65 & 2.7 & 347 & 40 & 0.8 \\
\hline- & - & 383 & 62 & 2.4 & 355 & 43 & 2.4 \\
\hline
\end{tabular}

spond to each other, but smallest if all but one are absent, as in the present case.

Though face orientation had a strong impact on stimulus coding, it did not affect response selection directly, as witnessed by the absence of an effect of orientationresponse correspondence. However, although this null effect with $90^{\circ}$ tilting was replicated in Experiment 2, we also found that some interaction of orientation and response location can be obtained with $45^{\circ}$ tilting (as will be discussed later).

The absence of a significant mapping effect is somewhat surprising from the salient-feature approach advocated by Weeks and Proctor (1990). They assume that S-R translation is faster with correspondence of the salient (and nonsalient) features of stimulus and response sets. Since right positions are thought to be more salient than left, and upper positions more salient than lower, the mapping of top stimulus-right response and bottom stimulus-left response should yield faster RTs than the reverse mapping. However, it should be noted that, while such a mapping effect occurred in some experiments (Dutta \& Proctor, 1992; Michaels, 1989; Weeks \& Proctor, 1990, Experiment 3), it did not occur in others (Bauer \& Miller, 1982; Michaels \& Schilder, 1991; Weeks \& Proctor, 1990, Experiment 1). That is, our failure to find a mapping effect is not extraordinaryand thus not necessarily of theoretical importance- the more so as a numerical advantage for the top-right/ bottom-left mapping is present even in our data.

\section{EXPERIMENT 2}

Experiment 1 has shown that, in an S-R compatibility task, the coding of stimulus position depends on context information. Since, in this task, position was the relevant stimulus feature, it is open to question whether context-dependent spatial coding is due to a strategy or whether it is an automatic process. Possibly, contextdefined position codes are formed only if the task requires some kind of spatial stimulus coding. In Experiment 2 , we used a Simon task where only the brightness of the stimulus mattered but not its position: Subjects pressed a left or right key in response to a black or white solid circle, respectively. Again, the stimuli appeared either on one or on the other eye. Since the Simon task per- mits an instruction-independent manipulation of stimulus position, face orientation was varied over a wider range: upright or tilted $90^{\circ}$ or $45^{\circ}$ to the left or right.

If context-dependent spatial stimulus coding were fully automatic, we would expect an eye-response correspondence effect for all orientations. If, however, contextrelative coding depended on the task relevance of stimulus position, no $\mathrm{S}-\mathrm{R}$ compatibility effect should be obtained with $90^{\circ}$ tilting - hence, with a physically orthogonal spatial S-R relationship.

\section{Method}

Twenty paid adults who fulfilled the same criteria as in Experiment 1 participated in single sessions of about $25 \mathrm{~min}$. Apparatus and procedure were as in Experiment 1 with the following exceptions: The experiment was controlled by a Rhotron VME system. The context stimulus was the same image, but there were five orientation conditions with the face appearing upright, or tilted $90^{\circ}$ or $45^{\circ}$ to the left or right. Irrespective of face orientation, the facerelative locations of fixation cross and stimuli were as in Experiment 1 . That is, the fixation cross always appeared at the center of the face (and of the display), and the stimuli (black or white solid circles of $1^{\circ}$ in diameter) appeared $0.9^{\circ}$ away from it, covering the left or the right eye, respectively.

The subjects responded to the white and black circles by pressing the left and the right key, respectively. Stimuli were introduced as "white" and "black," and it was emphasized that stimulus location would be irrelevant. At the beginning of the experiment, the subjects were familiarized with the five face orientations by presenting these in a clockwise order from $90^{\circ}$ tilted left to $90^{\circ}$ tilted right. With each face, the subjects performed a four-trial miniblock consisting of the possible combinations of two stimulus positions and two response locations in random order. In the following experimental phase, there were seven blocks of 40 trials each. In each block, the five face orientations were presented in random succession. Each face orientation remained constant throughout eight consecutive trials that consisted of two four-trial miniblocks.

\section{Results and Discussion}

Missing trials $(<0.5 \%)$ were excluded. Mean RTs and PEs were calculated as a function of degree of tilting, eye-response correspondence, and orientation-response correspondence (see Table 2), so that each RT mean of each subject was based on 28 valid observations. Since orientation-response correspondence was not defined for the upright condition, only the four tilting conditions were considered in the analysis. PEs were analyzed, but there was again not one significant result. 
Table 2

Mean Reaction Times (RTs, in Milliseconds), Standard Deviations (SDs), and Percent Errors (PEs) in Experiment 2 as a Function of Degree of Tilting, Orientation-Response Correspondence (ORC), and Eye-Response Correspondence (ERC)

\begin{tabular}{|c|c|c|c|c|c|c|c|c|c|c|}
\hline \multirow[b]{2}{*}{ ORC } & \multirow[b]{2}{*}{ ERC } & \multicolumn{3}{|c|}{$0^{\circ}$} & \multicolumn{3}{|c|}{$45^{\circ}$} & \multicolumn{3}{|c|}{$90^{\circ}$} \\
\hline & & RT & $S D$ & $\mathrm{PE}$ & RT & $S D$ & PE & RT & $S D$ & $\mathrm{PE}$ \\
\hline+ & + & & & & 466 & 71 & 4.0 & 469 & 70 & 3.0 \\
\hline+ & - & & & & 484 & 58 & 3.7 & 472 & 59 & 3.0 \\
\hline- & + & 462 & 76 & 2.9 & 481 & 81 & 3.8 & 459 & 63 & 2.6 \\
\hline- & - & 490 & 65 & 4.0 & 497 & 73 & 3.8 & 471 & 62 & 3.8 \\
\hline
\end{tabular}

A three-way ANOVA of the RTs showed a significant main effect of eye-response correspondence $[F(1,19)=$ $8.17, p<.01]$, confirming that responses were $12 \mathrm{msec}$ faster if their relative position corresponded to the facerelative position of the stimulus than if not ( 469 vs. $481 \mathrm{msec}$ ). This effect was not modified by degree of tilting or orientation-response correspondence $(p>.18$ and $p>.4$, respectively). So, context-dependent spatial stimulus codes were formed and affected performance, although stimulus position was completely irrelevant for the task. This not only provides additional support for the idea of context-dependent spatial stimulus coding but further suggests that the underlying coding process runs off automatically. However, it should also be noted that there was a numerical decrease of the eye-response correspondence effect from 17 to $8 \mathrm{msec}$ with increasing degree of tilting (see also the 28 -msec effect with $0^{\circ}$ ). Though this tendency was not reliable, it does indicate that the absence of retinal, egocentrical, and environmental left-right cues may indeed yield some reduction of the overall effect, just as assumed in Experiment 1.

Furthermore, there was a main effect of degree of tilting $[F(1,19)=15.80, p<.001]$ that entered into an interaction with orientation-response correspondence $[F(1,19)=9.52, p<.01]$ : Responses were faster with orientation-response correspondence than with noncorrespondence in the $45^{\circ}$ condition ( $475 \mathrm{vs.} 489 \mathrm{msec}$ ), but not in the $90^{\circ}$ condition ( $470 \mathrm{vs.} 465 \mathrm{msec}$ ), where the effect was numerically inverted. This result fits well to the null effect of orientation-response correspondence in Experiment 1, where only $90^{\circ}$ tilted faces were used. However, the present $45^{\circ}$ condition demonstrates a novel correspondence effect that is related not to stimulus location but to the orientation of the context. The finding that this effect is independent from, and, hence, additive to, the S-R location correspondence effect is consistent with the claim of multiple spatial coding. Obviously, both stimulus location and context orientation are coded and facilitate or interfere with selecting spatially defined responses. We can only speculate why the contextrelated effect could be observed with $45^{\circ}$ only but not with $90^{\circ}$ : Perhaps, small deviations of a face's (or object's) vertical main axis from the egocentric or retinal vertical generally yield the perception of directional (i.e., leftward or rightward) tilting, whereas a $90^{\circ}$ deviation gives the impression of an orthogonally situated (i.e., lying) face or object.

\section{GENERAL DISCUSSION}

Experiments 1 and 2 yielded two main results: First, visual stimuli presented above and below a fixation point are coded as left or right if the visual context induces such an interpretation, thus facilitating responses with a corresponding relative position (and/or interfering with noncorresponding responses). Second, context-induced coding does not depend on stimulus location being relevant to the task but also occurs in a Simon-type task. That is, context-relative coding seems to occur independently from the subject's intention-hence, automatically.

These results are consistent with demonstrations that subjects can use different reference frames for coding stimuli and responses in a compatibility task (Ladavas \& Moscovitch, 1984; Lippa, 1995). Beyond that, they clearly show that spatial stimulus coding is not confined to retinal, egocentrical, or environmental reference frames but also occurs in reference to a visual context that allows for object-based, intrinsic positional coding (Hinton \& Parsons, 1981; Palmer, 1989; Rock, 1973). Because such a coding can be assumed to be computationally expensive (Baylis \& Driver, 1993; Corballis, 1988), the finding that object-based codes are formed even if not required by the task suggests a high degree of automaticity in spatial stimulus analysis.

Our results add to, and are fully consistent with, prior demonstrations of multiple spatial stimulus coding, such as provided by Umiltà and Liotti (1987) and Lamberts et al. (1992). Interestingly, there is evidence that responses are also cognitively represented by several, probably simultaneously formed, spatial codes referring to the action goal, the effector location, and the anatomical mapping of the effector (Bradshaw et al., 1994; Hommel, 1993; Nicoletti, Umiltà, \& Ladavas, 1984; Riggio et al., 1986). That is, automatic, multiple spatial coding seems to be a general phenomenon in both stimulus and response coding.

This evidence for multiple spatial coding (whether within or between trials) casts some doubt on approaches claiming that only a single spatial stimulus code is formed. For instance, Stoffer (1991) or Umiltà and Nicoletti (1992) propose that such a code is formed only if a shift of spatial attention is prepared, a process that is assumed to be identical with programming an eye movement. If (plans for) eye movements were involved, the spatial code should refer to a retinal reference system or some equivalent. That is, spatial compatibility effects should always and only refer to retinal location, and this is not consistent with the present findings.

Finally, the results of the present experiments, as well as those of other studies, strongly suggest that cognitive codes and the subject's interpretation(s) of the situation play an important role in the emergence of compatibility phenomena. Though this view is not always shared (see Michaels, 1993; see also Proctor, Lu, Van Zandt, \& Weeks, 1994, for a rejoinder), it is supported by a growing body of converging evidence.

\section{REFERENCES}

BAUER, D. W., \& Miller, J. (1982). Stimulus-response compatibility and the motor system. Quarterly Journal of Experimental Psychology, 34A, 367-380.

BAYLIS, G. C., \& Driver, J. (1993). Visual attention and objects: Evidence for hierarchical coding of location. Journal of Experimental Psychology: Human Perception \& Performance, 19, 451-470.

Bradshaw, J. L., Willmott, C. J., Umiltà, C., Phillips, J. G., BradSHAW, J. A., \& MATTINGLEY, J. B. (1994). Hand-hemispace spatial 
compatibility, precueing, and stimulus-onset asynchrony. Psychological Research/Psychologische Forschung, 56, 170-178.

Broadbent, D. E., \& Gregory, M. (1962). Donders' b- and creactions and S-R compatibility. Journal of Experimental Psychology, 63, 575-578.

Corballis, M. C. (1988). Recognition of disoriented shapes. Psychological Review, 95, 115-123.

Dutta, A., \& Proctor, R. W. (1992). Persistence of stimulus-response compatibility effects with extended practice. Journal of Experimen. tal Psychology: Learning, Memory, \& Cognition, 18, 801-809.

Hinton, G. E., \& Parsons, L. M. (1981). Frames of reference and mental imagery. In J. Long \& A. Baddeley (Eds.), Attention and performance IX (pp. 261-277). Hillsdale, NJ: Erlbaum.

HOMMEL, B. (1993). Inverting the Simon effect by intention: Determinants of direction and extent of effects of irrelevant spatial information. Psychological Research/Psychologische Forschung, 55, 270-279.

Ladavas, E., \& Moscovitch, M. (1984). Must egocentric and environmental frames of reference be aligned to produce spatial $S-R$ compatibility effects? Journal of Experimental Psychology: Human Perception \& Performance, 10, 205-215.

LamberTs, K., TAVERnier, G., \& D'Ydewalle, G. (1992). Effects of multiple reference points in spatial stimulus-response compatibility. Acta Psychologica, 79, 115-130.

LIPPA, Y. (1995). A referential-coding explanation for compatibility effects of physically orthogonal stimulus and response dimensions. Manuscript submitted for publication

MiChaELS, C. F. (1989). S-R compatibilities depend on eccentricity of responding hand. Quarterly Journal of Experimental Psychology, 41A, 263-272.

Michaels, C. F. (1993). Destination compatibility, affordances, and coding rules: A reply to Proctor, Van Zandt, Lu, and Weeks (1993). Journal of Experimental Psychology: Human Perception \& Performance, 19, 1121-1127.

Michaels, C. F., \& SchiLder, S. (1991). Stimulus-response compatibilities between vertically oriented stimuli and horizontally oriented responses: The effects of hand position and posture. Perception \& Psychophysics, 49, 342-348.

Nicoletti, R., Umiltà, C., \& Ladavas, E. (1984). Compatibility due to the coding of the relative position of the effectors. Acta Psychologica, 57, 133-143.

PALMER, S. E. (1989). Reference frames in the perception of shape and orientation. In B. E. Shepp \& S. Ballesteros (Eds.), Object perception: Structure and process (pp. 121-163). Hillsdale, NJ: Erlbaum.

Proctor, R. W., Lu, C.-H., VAn ZANDT, T., \& Weeks, D. J. (1994). Affordances, codes, and decision processes: A response to Michaels (1993). Journal of Experimental Psychology: Human Perception \& Performance, 20, 452-455.

Riggio, L., GaWryszewski, L. G., \& UMiLtà, C. (1986). What is crossed in crossed-hand effects? Acta Psychologica, 62, 89-100. Rock, I. (1973). Orientation and form. New York: Academic Press.

Simon, J. R., HinRIChs, J. V., \& CRAFT, J. L. (1970). Auditory S-R compatibility: Reaction time as a function of ear-hand correspondence and ear-response-location correspondence. Journal of Experimental Psychology, 86, 97-102.

StOFFER, T. H. (1991). Attentional focussing and spatial stimulusresponse compatibility. Psychological Research/Psychologische Forschung, 53, 127-135.

UMiltà, C., \& LIOTTI, M. (1987). Egocentric and relative spatial codes in S-R compatibility. Psychological Research, 49, 81-90.

UMilis̀, C., \& Nicoletti, R. (1992). An integrated model of the Simon effect. In J. Alegria, D. Holender, J. Junça de Morais, \& M. Radeau (Eds.), Analytic approaches to human cognition (pp. 331-350). Amsterdam: Elsevier.

WeEKS, D. J., \& Proctor, R. W. (1990). Salient-features coding in the translation between orthogonal stimulus and response dimensions. Journal of Experimental Psychology: General, 119, 355-366.

\section{NOTES}

1. Instead of multiple coding, one may assume that only one code at a time is formed, with the reference frame varying (randomly?) from trial to trial. However, in the absence of conclusive empirical data on that issue, this seems to be the more arbitrary and less parsimonious theoretical alternative.

2. This kind of context stimulus was used (1) because the mirror symmetry of faces suggests a natural coding of parts of the face in terms of left and right, and (2) because pilot studies showed less consistent effects with less structured face drawings.

(Manuscript received July 28, 1994; revision accepted for publication January 3,1995 .) 